\title{
New Supraventricular Tachycardia Initiative Seeks to Help Patients Find the Best Possible Care and Support
}

\author{
Hugh Calkins \\ Johns Hopkins Medical Institutions, Baltimore, MD, USA
}

DOI: https://doi.org/10.17925/EJAE.2021.7.1.2

$\mathrm{T}$ he charity Arrhythmia Alliance has launched a new initiative - a supraventricular tachycardia (SVT) Centres of Excellence programme - to help people with SVT to find the best possible care and support for their condition. As part of the programme, the Arrhythmia Alliance has also published the first annual SVT Pioneers Report, which explores new and innovative approaches to managing SVT. This editorial explains how the new initiative, including the report, encourages healthcare professionals and patients to collaborate to identify optimal SVT care.

\section{Keywords}

Supraventricular tachycardia, arrhythmia, Arrhythmia Alliance, Centre of

Excellence, SVT Pioneers Report

Disclosure: Hugh Calkins has received speaker honoraria and consultant fees from Atricure, Biosense Webster and Medtronic.

Acknowledgements: Editorial and writing assistance was provided by Dawn Powell of the Arrhythmia Alliance and Mrs Trudie C A Lobban, MBE, Founder and CEO of the Arrhythmia Alliance.

Review process: Double-blind peer review

Compliance with ethics: This article is an opinion piece and does not report on new clinical data, or any studies with human or animal subjects performed by any of the authors.

Authorship: The named author meets the criteria of the International Committee of Medical Journal Editors for authorship for this manuscript, takes responsibility for the integrity of the work as a whole and has given final approval for the version to be published.

Access: This article is freely accessible at touchCARDIO.com (C) Touch Medical Media 2021.

Received: 22 April 2021

Accepted: 20 May 2021

Published Online: 7 June 2021

Citation: European Journal of Arrhythmia \& Electrophysiology. 2021;7(1):2-3

Corresponding author: Hugh Calkins,

Johns Hopkins Medical Institutions, 600 N Wolfe St.

Baltimore, MD 21287, USA.

E:hcalkins@jhmi.edu

Support: No funding was received for

the publication of this article.
Supraventricular tachycardia (SVT) is, as its name implies, a tachycardia or elevated heart rate (more than 100 beats per minute) that originates above the ventricles (i.e. in the atria). Or, to use the specific terminology of the European Society of Cardiology (ESC), SVT "literally indicates tachycardia, the mechanism of which involves tissue from the His bundle or above". ${ }^{\text {Go }}$ et al. report that, in the USA, "the incidence of presumed new symptomatic paroxysmal SVT (PSVT) episodes between 2010 and 2015 was 73 per 100,000". ${ }^{2}$ They add that the prevalence of PSVT is "substantially higher with older age" with "a $>20$-fold higher prevalence for participants aged $\geq 65$ years compared with those aged 18 to 24 years"; ${ }^{\prime 2}$ however, according to the ESC, Ione PSVT, opposed to PSVT with cardiovascular disease, is more common among younger people (note, the use of SVT versus PSVT reflects the terminology used in the original papers). Furthermore, the ESC states that people with lone PSVT have "an earlier onset of symptoms and are more likely to have their condition first documented in the emergency department". ${ }^{1}$

The symptoms of SVT, as with many arrhythmias, depend on the individual characteristics of the person with SVT, and can range from palpitations to syncope. However, Kotadia et al. observe that SVT increases patient morbidity "particularly when symptoms are frequent or incessant, and in a small cohort of patients with atrial fibrillation and ventricular pre-excitation, it can be life-threatening". ${ }^{~}$ Furthermore, they note: "SVTs are a common cause of presentation to primary and secondary care services and can cause significant patient discomfort and distress. ${ }^{\text {"3 }}$ Similarly, Go et al. found that about 50,000 visits to emergency departments in the USA are connected with PSVT. ${ }^{2}$

The ESC state that, for the diagnosis of SVT, an electrocardiogram recorded during an episode of SVT is ideal, and people experiencing symptoms of SVT should "be encouraged to seek medical assistance and a recording of their ECG during episodes". ${ }^{.}$However, they acknowledge that the episodic nature of the condition means that recording an electrocardiogram (ECG) at the time of tachycardia can be difficult.

In the current climate of the COVID-19 pandemic, this has become even more challenging. In the new publication "Personal ECG devices - a useful adjunctive tool to diagnose SVTs", Haldar observes that while people with SVT continued to present at his centre during the first few months of the pandemic (Royal Brompton and Harefield Hospitals, London, UK), "many probably have not sought help for fear of attending hospitals and the potential for contracting the coronavirus". ${ }^{4}$ Mindful that patients may want to avoid hospital, and of the need to reduce in-person footfall to the hospital, Haldar and his colleagues "sent many patients ECG recording monitors with video guidance on how to fit/wear them". He adds that they also encouraged the use of mobile ECG devices, such as the KardiaMobile (Alivecor, Mountain View, CA, USA) device or the Apple Watch (Apple, Los Altos, CA, USA). According to Haldar, the use of these devices helped to improve the diagnosis of SVT during the pandemic - to the extent that he believes such tools are now here to stay. "Ultimately, having wider options and embracing the potential of digital health tools is likely to be beneficial for patients and physicians alike with hopefully shorter times to diagnosis and treatment", he concludes. 
Piccini, in his case study for the "SVT Pioneers Report", also advocates the potential of mobile ECG devices to aid with diagnosis and management of SVT. ${ }^{5}$ He states: "Further evolution of wearable technologies may make 12-lead electrocardiograms easier to obtain and may further improve the diagnosis of SVT".

The aim of the SVT Pioneers Report is to provide examples of good care to inspire healthcare professionals, and arrhythmia centres, to consider what innovative approaches they could adopt to improve care and quality of life for people with SVT. In fact, the report is part of a new initiative - the Arrhythmia Alliance SVT Centres of Excellence programme - to develop an online resource (www.SVTPioneers.org) to help both patients and healthcare professionals to identify the best possible care for people with SVT.

For example, by using the resource's Centre of Excellence pages, patients can locate centres near them that have expertise in managing SVT. Healthcare professionals who are not arrhythmia specialists can also use the pages to find centres that they can refer patients to. As all the centres listed on the pages have been vetted by the Arrhythmia Alliance, patients and healthcare professionals alike can be safe in the knowledge they are contacting centres that have the relevant knowledge and skills in managing SVT.

To be accredited as an Arrhythmia Alliance SVT Centre of Excellence, centres give details of the services that they provide and the specialists who work there. An Arrhythmia Alliance expert panel of judges, chaired by myself in my capacity as Medical Director and Board Member of Arrhythmia Alliance US, then review these details to determine if a centre should be added to the Centre of Excellence pages. The panel look for centres that are providing specific services for people with SVT and for centres that have specialists with experience in managing SVT.

Centres can also be listed as a Centre of Excellence by submitting a case study for future editions of the "SVT Pioneers Report"; any centre with an accepted case study is automatically listed on the Centres of Excellence pages. As assessed by the Arrhythmia Alliance expert panel of judges, accepted case studies should demonstrate a novel or innovative approach to managing SVT - and, importantly, an approach that takes account of the individual needs of the person with SVT. The Arrhythmia Alliance hopes that, with the "SVT Pioneers Report", centres are not only inspired to adopt innovative approaches to managing people with SVT, but are also inspired to share those approaches via the Arrhythmia Alliance SVT Centres of Excellence programme, so, they, in turn, can encourage others to become SVT pioneers and Centres of Excellence. With the programme still in its infancy, at this time we are encouraging people to read the report and apply to become a Centre of Excellence; but in time, we hope to develop ways to objectively assess the value of this initiative.

Of note, the SVT Centres of Excellence programme specifically focuses on PSVT, as with the ESC SVT guidelines. The ESC report that their SVT guidelines do not cover atrial fibrillation (AF) because AF "is the subject of separate clinical Guidelines and various consensus documents"." Similarly, the SVT Centres of Excellence programme does not cover AF, as the AF Association, sister organization to Arrhythmia Alliance, already publishes the annual "AF Association Healthcare Pioneers Report Showcasing Best Practice in $\mathrm{AF}^{\prime \prime}{ }^{6}$ Furthermore, the AF Association is in the process of developing an AF Centres of Excellence programme.

Collaboration, with patients as well as between different healthcare specialists, is key to the optimal management of any condition, including SVT. Therefore, by working together, arrhythmia specialists, non-arrhythmia specialists and patients can help to create a new online resource that, with time, will become a truly global portal for finding the best possible care for SVT. The SVT Centres of Excellence programme, including the "SVT Pioneers Report", is not meant as a replacement for research published in journals or presented at congresses, but rather as a tool for sparking ideas about what could be done to improve care. Therefore, this collaboration and discussion may eventually help to address the current challenges of SVT. $\square$

. Brugada J, Katritsis DG, Arbelo E, et al. 2019 ESC Guidelines for the management of patients with supraventricular tachycardia. Eur Heart J. 2020;41:655-720.

Go AS, Hlatky MA, Liu TI, et al. Contemporary burden and correlates of symptomatic paroxysmal supraventricular tachycardia. J Am Heart AsSOC. 2018;7:0008759.

Kotadia ID, Williams SE, O'Neil M. Supraventricular tachycardia: An overview of diagnosis and management. Clin Med (Lond). 2020;20:43-7.

4. Haldar S. Personal ECG devices - a useful adjunctive tool to diagnose SVTs. Available at: www.heartrhythmalliance.org/aa/us/healthcare-pioneers-report/case-studies/31 (accessed 21 May 2021).

Piccini J. Bigeminy, aberrancy, and atrial tachycardia in a patient with palpitations: The Importance of 12-lead electrocardiography. Arrhythmia Alliance Healthcare Pioneers Report — Showcasing Best Practice in SVT. 2021. Available at: www.heartrhythmalliance.org/aa/us/healthcare-pioneers-report/case-studies/35 (accessed 27 May 2021).

6. AF Association. AF Association Healthcare Pioneers Report - Showcasing Best Practice in AF 2021. Available at: www.heartrhythmalliance.org/files/files/AFA\%20Healthcare\%20Pioneers.pdf (accessed 27 May 2021). 